\title{
CRITICAL GROUPS AND THE LATTICE OF VARIETIES
}

\section{JOHN COSSEY}

1. Introduction. The set of all varieties of groups, partially ordered by inclusion, forms a complete lattice: this lattice is always modular, for it is the dual of the lattice of verbal subgroups of the absolutely free group of countably infinite rank (for this and other basic results about varieties of groups, we refer the reader to [8]). However, the lattice is not in general distributive: the first example of a nondistributive lattice is due to Graham Higman, who showed that the lattice of varieties of groups of exponent $p$ and class less than $p$ is not distributive for sufficiently large primes $p$ [8, Theorem 54.2]. L. G. Kovács and M. F. Newman [7] have shown that even the lattice of metabelian varieties is not distributive. In contrast, we exhibit in this paper a lattice of varieties which is distributive.

To state our results, we need some notation. If $N$ is a normal subgroup of a subgroup $H$ of a group $G$, we call $H / N$ a factor of $G$ : if $N \neq 1$, or $H \neq G$, we call $H / N$ a proper factor of $G$. A group $G$ is called critical if it is finite and not contained in the variety generated by its proper factors. If $G$ is critical, it is easily seen that $G$ has a unique minimal normal subgroup, which is called the monolith of $G$ and denoted by $\sigma G$. We define an $A^{*}$-group to be a finite group all of whose Sylow subgroups are abelian, and whose composition factors are known simple groups. (In fact, all finite simple groups all of whose Sylow subgroups are abelian are known: the assumption is kept here since the result is as yet unpublished.) A variety of $A^{*}$-groups will be a locally finite variety whose finitely generated groups are $A^{*}$-groups.

The main result of this paper is

\section{TheOREM 1. The lattice of varieties of $A^{*}$-groups is distributive.}

This theorem is a consequence of the following two theorems:

Theorem 2 (L. G. Kovács and M. F. Newman [5]). If $G$ is a critical group with nonabelian monolith, and $D$ is a class of groups such that var $\mathbb{D}$ (the variety generated by $\mathbb{D}$ ) is locally finite, then $G \in \operatorname{var} D$ if and only if $G$ is isomorphic to a factor of some $D \in D$.

THEOREM 3. If $G$ is a critical $A^{*}$-group with abelian monolith, and $D$ is a class of groups such that $\operatorname{var} D$ is a variety of $A^{*}$-groups, then $G \in \operatorname{var} D$ if and only if $G$ is isomorphic to a factor of some $D \in D$.

Received by the editors June 19, 1967. 
It is tempting to hope that the condition of Theorem 1 that $D$ generate a variety of $A^{*}$-groups can be relaxed to the condition that $D$ generate a locally finite variety. However, L. G. Kovács [4] has constructed examples which show this hope is in vain.

Most of the work for this paper was done while I held a Postgraduate Research Scholarship at the Australian National University. I thank Dr. L. G. Kovács, under whose supervision this work was done.

2. Notation and preliminary results. For the rest of this paper, groups are finite unless otherwise indicated. The subgroup of a group $G$ generated by all the minimal normal subgroups of $G$ is called the socle of $G$, and denoted by $\sigma G$ : the centralizer of $\sigma G$ in $G$ is denoted by $\sigma^{*} G$. A group is called homocyclic if it is the direct product of isomorphic cyclic groups of prime power order. A normal homocyclic subgroup $N$ of a group $G$ is called indecomposable if $N$ contains no proper subgroup of the same exponent normal in $G$. The subgroup of a group $G$ generated by the $n$th powers of the elements of $G$ is denoted by $G^{n}$. If $S$ is a subset of a group $G$, then $C_{G}(S)$ denotes the centralizer of $S$ in $G$.

For soluble $A^{*}$-groups, the following lemma is due to D. R. Taunt [10, Theorem 4.1], the result is presumably well known, but does not seem to occur in the literature. We sketch a proof.

Lemma 1. If $G$ is an $A^{*}$-group, then $Z(G) \cap G^{\prime}=1$ (where $Z(G)$ is the center of $G$ ).

Proof. Suppose that the result is not true, and let $G$ be a minimal counterexample. It is easy to see that $Z(G) \cap G^{\prime}$ is a $p$-group for some prime $p$, and is the unique minimal normal subgroup of $G$. Transfer $G$ into a Sylow $p$-subgroup of $G$; an easy computation shows that $Z(G) \cap G^{\prime}$ cannot be in the kernel of this homomorphism, and so the transfer is an isomorphism. But then $G$ is abelian, and the lemma is true for abelian groups, a contradiction.

Lemma 2. Let $G$ be a critical $A^{*}$-group with abelian monolith. Then $\sigma G$ is a p-group for some prime $p$, and if $G_{p}$ is a Sylow p-subgroup of $G, G_{p}=\sigma^{*} G$. Further, $\sigma^{*} G$ is an indecomposable normal homocyclic subgroup of $G$.

Proof. Since $\sigma G$ is abelian, it can be written as the direct product of its Sylow subgroups, each of which is characteristic in $\sigma G$ and hence normal in $G$. But $G$ is critical and thus $\sigma G$ is a $p$-group. If $\left(\sigma^{*} G\right)^{\prime} \neq 1$, it contains $\sigma G$ : also, since $\sigma G$ is abelian, $\sigma G \leqq \sigma^{*} G$, and so $\sigma G \leqq Z\left(\sigma^{*} G\right)$ and we may conclude from Lemma 1 that $\sigma^{*} G$ is abelian. As for $\sigma G$, 
we conclude $\sigma^{*} G$ is a $p$-group. Hence, if $G_{p}$ is a Sylow $p$-subgroup, we have $G_{p} \leqq \sigma^{*} G \leqq G_{p}$; i.e. $\sigma^{*} G=G_{p}$.

Now $\sigma\left(\sigma^{*} G\right)$ is normal in $G$; being an elementary abelian p-group, we may regard it as a vector space over the field of $p$ elements. Maschke's theorem then gives us that it is completely reducible as a $G$-module. Hence $\sigma G=\sigma\left(\sigma^{*} G\right)$. It now follows easily that $\sigma^{*} G$ is an indecomposable normal homocyclic subgroup of $G$.

We will need the idea of similarity of minimal normal subgroups, introduced by L. C. Kovács and M. F. Newman in [5]. Suppose that $M$ is a minimal normal subgroup of $G, N$ a minimal normal subgroup of $H$ : then we say $M$ (qua normal subgroup of $G$ ) is similar to $N$ (qua normal subgroup of $H$ ) if there exist isomorphisms $\mu: M \rightarrow N$ and $\nu: G / C_{G}(M) \rightarrow H / C_{H}(N)$ such that, for $x \in M, h \in G$,

$$
\left(x^{h C_{G}(M)}\right) \mu=(x \mu)^{\left(h C_{G}(M)\right) \nu} .
$$

We write $M \sim N$

Lemma 3. If $G, H$ are critical $A^{*}$-groups with abelian monoliths such that $\sigma G \sim \sigma H$ and $\sigma^{*} G, \sigma^{*} H$ have the same exponent, then $G \cong H$.

Proof. We may conclude from the Schur-Zassenhaus theorem and Lemma 2 that $\sigma^{*} G$ is complemented in $G$, and so $G$ is determined by the action of $G / \sigma^{*} G$ on $\sigma^{*} G$. Since inner automorphisms commute with the power map $x \rightarrow x^{p^{\alpha}}, x \in \sigma^{*} G, p|| \sigma G \mid$, we have that $\sigma G$ (qua normal subgroup of $G$ ) is similar to $\sigma^{*} G /\left(\sigma^{*} G\right)^{p}$ (qua normal subgroup of $G /\left(\sigma^{*} G\right)^{p}$ ): also $\sigma H$ (qua normal subgroup of $H$ ) is similar to $\sigma^{*} H /\left(\sigma^{*} H\right)^{p}$ (qua normal subgroup of $H /\left(\sigma^{*} H\right)^{p}$ ). Then, the relation of similarity being transitive, we have

$$
\sigma^{*} G /\left(\sigma^{*} G\right)^{p} \sim \sigma^{*} H /\left(\sigma^{*} H\right)^{p} .
$$

But from this it follows, using a theorem of Philip Hall [1, Theorem 12.2.2] that the action of $G / \sigma^{*} G$ on $\sigma^{*} G$ is the same as the action of $H / \sigma^{*} H$ on $\sigma^{*} H$, and hence $G \cong H$.

3. The proof of Theorem 3. We start by observing that if $G \in \operatorname{var} D$, we can assume that $D$ is a finite set of finite groups, by Lemma 4.2 of Higman [2]. Let $D^{*}$ denote the factor closure of $D$, i.e. a group is in $D^{*}$ if and only if it is isomorphic to a factor of some $D \in D$. Then there exist $D_{i} \in D^{*}, 1 \leqq i \leqq n$, such that $G$ is isomorphic to a factor of $\prod_{i=1}^{n} D_{i}$, and

(1) $D_{i}$ is critical, $1 \leqq i \leqq n[6,1.11]$,

(2) $\sigma G \sim \sigma D_{i}, 1 \leqq i \leqq n[6,1.12]$,

(3) $\sigma^{*} G \in \operatorname{var} \prod_{i=1}^{n} \sigma^{*} D_{i}[6,1.14]$. 
From the definition of similarity, (2) implies that $D_{i}$ has an abelian monolith, and so, being a critical $A^{*}$-group, $\sigma^{*} D_{i}$ is an indecomposable normal homocyclic subgroup of $D_{i}$, and if $p|| \sigma G \mid, \sigma^{*} D_{i}$ is the Sylow $p$-subgroup of $D_{i}, 1 \leqq i \leqq n$ (Lemma 2). By the Schur-Zassenhaus theorem, $\sigma^{*} G$ has a complement $H$ in $G$, and $\sigma^{*} D_{i}$ has a complement $K_{i}$ in $D_{i}$; then (2) tells us that $H \cong K_{i}, 1 \leqq i \leqq n$. Also, if $\sigma^{*} G$ has exponent $p^{l}$, (3) implies that at least one $\sigma^{*} D_{i}$ has exponent $p^{k}$, with $k \geqq l$; suppose $\sigma^{*} D_{1}$ has. Put $D=D_{1} / N$, where $N=\left(\sigma^{*} D_{1}\right)^{p l}$. Observe that since inner automorphisms commute with the power map $x \rightarrow x^{p l}$, $x \in \sigma^{*} D_{1}$, we have $\sigma D \sim \sigma G$. Since $\sigma^{*} G$ and $\sigma^{*} D$ have the same exponent, $G \cong D$ by Lemma 3 ; i.e. $G \in D^{*}$.

The other direction is obvious.

4. The lattice of varieties of $A^{*}$-groups. We take a complete set $a$ of pairwise nonisomorphic critical $A^{*}$-groups, and partially order this set by defining $A-B$ if and only if $A$ is isomorphic to a factor of $B, A, B \in Q$. It is convenient here to include the trivial group in $a$, though it is not usually considered a critical group. We define an ideal $\mathscr{L}$ in $a$ to be a subset of $a$ such that if $B \in \mathscr{L}$ and $A-<B$, then $A \in \mathcal{L}$. An abelian element of $a$ has the obvious meaning: we call an ideal small if it contains only a finite number of abelian elements. It is easy to check that the set of small ideals of $a$ forms a lattice with respect to partial order by inclusion.

We show that the lattice of varieties of $A^{*}$-groups is isomorphic to this lattice. To be specific, we claim that the map

$$
\phi: \mathfrak{L} \rightarrow \operatorname{var} \mathfrak{L}
$$

is a lattice isomorphism. Since $\mathscr{L}$ contains only a finite number of abelian elements, there is an integer $e$ such that the groups in $\mathscr{L}$ have exponent dividing $e$.

The class of all $A^{*}$-groups of exponent dividing $e$ forms a locally finite variety [3, Theorem 4], and so is a variety of $A^{*}$-groups. Hence $\operatorname{var} \mathcal{L}$ is a variety of $A^{*}$-groups. It follows immediately from Theorems 2 and 3 that if $\mathfrak{L}_{1} \neq \mathscr{L}_{2}, \operatorname{var} \mathfrak{L}_{1} \neq \operatorname{var} \mathfrak{L}_{2}$. Also, if $\mathfrak{R}$ is a variety of $A^{*}$ groups, then $\& \cap Q$ is a small ideal. Thus $\phi$ is one to one and onto. If $\mathcal{L}$ and $\mathcal{R}$ are small ideals, then Theorems 2 and 3 imply that

$$
(\mathscr{L} \cap R) \phi=\mathscr{L} \phi \cap R \phi, \quad(\mathfrak{L} \cup R) \phi=\mathfrak{L} \phi \cup R \phi .
$$

Thus $\phi$ is an isomorphism.

We now have as a consequence of Theorem 83 of [9]

Theorem 3. The lattice of varieties of $A^{*}$-groups is distributive. 
We also have (the dual of Theorem 39 of [9]):

TheOREM 4. Every variety of $A^{*}$-groups can be written in at most one way as an irredundant union of join-irreducible subvarieties.

We remark that the question of whether every variety of $A^{*}$-groups can be written in at least one way is an open question, and presumably not an easy one, for it is not difficult to see that it is equivalent to the problem of whether there is a finite basis for the laws of every variety of $A^{*}$-groups.

\section{REFERENCES}

1. Marshall Hall, Jr., The theory of groups, Macmillan, New York, 1959.

2. Graham Higman, Some remarks on varieties of groups, Quart. J. Math. Oxford Ser. (2) 10 (1959), 165-178.

3. L. G. Kovács, Varieties and the Hall-Higman paper, Proc. Internat. Conf. Theory of Groups, Austral. Nat. Univ., Canberra, August 1965, Gordon and Breach, New York (to appear).

4. - unpublished.

5. L. G. Kovács and M. F. Newman, Minimal verbal subgroups, Proc. Cambridge Philos. Soc. 62 (1966), 347-350.

6. - On critical groups, J. Austral. Math. Soc. 6 (1966), 237-250.

7. —, unpublished.

8. Hanna Neumann, Varieties of groups, Ergebnisse der Mathematik und ihrer Grenzgebiete, Springer, Berlin, 1967.

9. Gabor Szasz, Introduction to lattice theory, Academic Press, New York, 1963.

10. D. R. Taunt, On A-groups, Proc. Cambridge Philos. Soc. 45 (1949), 24-42.

Graduate Center, The City University of New York 